\title{
GeV-scale dark matter: production at the Main Injector
}

\author{
Bogdan A. Dobrescu and Claudia Frugiuele \\ Theoretical Physics Department, Fermilab, \\ Batavia, IL 60510, U.S.A. \\ E-mail: bdob@fnal.gov, claudiaf@fnal.gov
}

ABSTRACT: Assuming that dark matter particles interact with quarks via a GeV-scale mediator, we study dark matter production in fixed target collisions. The ensuing signal in a neutrino near detector consists of neutral-current events with an energy distribution peaked at higher values than the neutrino background. We find that for a $Z^{\prime}$ boson of mass around a few $\mathrm{GeV}$ that decays to dark matter particles, the dark matter beam produced by the Main Injector at Fermilab allows the exploration of a range of values for the gauge coupling that currently satisfy all experimental constraints. The $\mathrm{NO} \nu \mathrm{A}$ near detector is well positioned for probing the presence of a dark matter beam, and future LBNF near detectors would provide more sensitive probes.

Keywords: Rare Decays, Beyond Standard Model, Neutrino Physics

ARXIV EPRINT: 1410.1566 


\section{Contents}

1 Introduction 1

2 Leptophobic $Z^{\prime}$ as portal to hidden particles 2

2.1 Limits from monojet searches 4

2.2 Invisibile quarkonium decays 4

2.3 Monophoton limits from BaBar data 4

2.4 Anomaly cancellation versus collider limits on fermions 4

3 Cosmological constraints on DM scenarios 5

$\begin{array}{lll}4 & \text { DM production through proton scattering off nucleons } & 7\end{array}$

5 DM flux through detectors $\quad 8$

5.1 Angular distribution of DM particles $\quad 9$

$\begin{array}{lll}5.2 & \text { Energy distribution of DM particles } & 10\end{array}$

$\begin{array}{lll}6 & \text { DM scattering inside the detector } & 11\end{array}$

$\begin{array}{lll}7 & \text { Outlook } & 14\end{array}$

\section{Introduction}

Dark matter (DM) provides solid evidence for physics beyond the Standard Model (SM), but its identity remains unknown. A central question is whether DM particles experience interactions with ordinary matter beyond gravity. If DM particles have weak-scale masses and order-one couplings, then their relic abundance given by the thermal freeze-out is consistent with the observed value [1]. An extensive experimental program has been carried out to explore this WIMP (weakly-interacting massive particles) paradigm, setting impressive bounds on the viable parameter space. Direct detection experiments [2], in particular, have imposed strong constraints on the interactions between nucleons and WIMPs of mass larger than about $5 \mathrm{GeV}$.

Here we consider the possibility that DM particles have mass below $5 \mathrm{GeV}$ and have interactions with quarks mediated by a new boson. If the latter is relatively light, then the DM interactions with nucleons can be probed in experiments. A natural choice for the mediator is a leptophobic $Z^{\prime}$ boson of mass near the GeV scale. The constraints on a boson of this type interacting with quarks are rather loose $[3,4]$. We will show that the constraints remain weak even when the $Z^{\prime}$ interacts with DM.

A promising search method for such light DM particles is to test if they are produced in fixed target experiments, which benefit from large beam intensities. We will focus on 
the case where the $Z^{\prime}$ boson decays into a pair of DM particles. Proton scattering off a fixed target may copiously produce $Z^{\prime}$ bosons of mass around a few $\mathrm{GeV}$ as long as the proton energy is larger than a few tens of GeV. Thus, the Main Injector [5] at Fermilab, which accelerates protons to $120 \mathrm{GeV}$, is well suited to test this $\mathrm{GeV}$-scale DM scenario. In the NuMi beam line, where the $120 \mathrm{GeV}$ protons hit a Carbon target, if DM particles are produced, then they could be detected as neutral-current events in neutrino near-detectors such as $\mathrm{NO} \nu \mathrm{A}[6,7]$ and MINOS [8, 9]. The future LBNF [10] beam line would be even better suited for this type of search, provided a detector is placed within a few hundred meters from the target.

The possibility of searching for dark matter beams at neutrino detectors has been recently proposed and explored in [11-14], especially for DM much lighter than $1 \mathrm{GeV}$, which can be produced in the Fermilab Booster beam line where the protons have an energy of $8 \mathrm{GeV}$ [15]. Hadron collider experiments are also sensitive to light DM that interacts with quarks, because a pair of DM particles could be produced in association with a jet or a photon or other particles [16-23]. Quarkonium decays that involve missing energy provide another probe of the GeV-scale DM scenario [24-30]. We are going to compare the existing limits from these classes of experiments, and show that DM beams produced at the Main Injector may lead to thousands of deep-inelastic neutral current events in existing and future neutrino detectors. This is an example of a broader capability of high-intensity fixed target experiments to probe the existence of light hidden particles [31-37].

\section{Leptophobic $Z^{\prime}$ as portal to hidden particles}

We are focusing on a vector boson $Z^{\prime}$ of mass $M_{Z^{\prime}}$ in the $1-10 \mathrm{GeV}$ range, so that it can be produced by an $O(100) \mathrm{GeV}$ proton beam scattering off a fixed target. To that end, we extend the SM gauge group by including an $\mathrm{U}(1)_{z}$ group under which the quarks are charged while the leptons are neutral. The simplest charge assignment that allows quark masses and evades constraints from flavor-changing neutral currents (FCNC) is charges given by the baryon number, $\mathrm{U}(1)_{B}$ [38-42]. Another possibility is to assign charge 0 to the left-handed quark doublets and to charge either the down- or the up-type right-handed quarks; a simple choice is $\mathrm{U}(1)_{d s}$, where $d_{R}$ and $s_{R}$ have charges +1 and -1 , while all other quarks are neutral [3]. Thus, the $Z^{\prime}$ boson has the following couplings to the SM quarks $q=u, d, s, c, b, t$,

$$
\mathcal{L}_{q}=\frac{g_{z}}{2} Z^{\prime \mu} \times \begin{cases}\frac{1}{3} \sum_{q} \bar{q} \gamma_{\mu} q, & \mathrm{U}(1)_{B} \text { case }, \\ \bar{d}_{R} \gamma_{\mu} d_{R}-\bar{s}_{R} \gamma^{\mu} s_{R}, & \mathrm{U}(1)_{d s} \text { case },\end{cases}
$$

Let us also include a very long lived particle, generically labelled by $\chi$, of mass $m_{\chi}<$ $M_{Z^{\prime}} / 2$, which is a color singlet, electrically neutral, but charged under $\mathrm{U}(1)_{z}$. We focus on the cases where $\chi$ is either a Dirac fermion or a complex scalar (possible DM scenarios are discussed in section 3). Occasionally we will use the notations $\psi_{\chi}$ or $\phi_{\chi}$ when we need to emphasize the difference between the fermion and scalar $\chi$. If $\chi$ is a Dirac fermion, then its left- and right-handed components may have different $\mathrm{U}(1)_{z}$ charges; for simplicity we 
will ignore this possibility though, and label the $\mathrm{U}(1)_{z}$ charge of $\chi$ by $z_{\chi}$ whether it is a fermion or a complex scalar. The $Z^{\prime}$ couplings to the long-lived particle $\chi$ are

$$
\mathcal{L}_{\chi}=\frac{g_{z}}{2} Z^{\prime \mu} \times\left\{\begin{array}{c}
z_{\chi} \bar{\psi}_{\chi} \gamma_{\mu} \psi_{\chi}, \quad \text { if Dirac fermion }, \\
i z_{\chi}\left[\left(\partial_{\mu} \phi_{\chi}^{\dagger}\right) \phi_{\chi}-\phi_{\chi}^{\dagger} \partial_{\mu} \phi_{\chi}\right], \text { if complex scalar },
\end{array}\right.
$$

The partial width for the $Z^{\prime}$ decay into a pair of $\chi$ particles is

$$
\Gamma\left(Z^{\prime} \rightarrow \psi_{\chi} \bar{\psi}_{\chi}\right)=\frac{g_{z}^{2} z_{\chi}^{2}}{48 \pi} M_{Z^{\prime}}\left(1+2 \frac{m_{\chi}^{2}}{M_{Z^{\prime}}^{2}}\right)\left(1-4 \frac{m_{\chi}^{2}}{M_{Z^{\prime}}^{2}}\right)^{1 / 2}
$$

for Dirac fermions, and

$$
\Gamma\left(Z^{\prime} \rightarrow \phi_{\chi}^{\dagger} \phi_{\chi}\right)=\frac{g_{z}^{2} z_{\chi}^{2}}{192 \pi} M_{Z^{\prime}}\left(1-4 \frac{m_{\chi}^{2}}{M_{Z^{\prime}}^{2}}\right)^{3 / 2} .
$$

for complex scalars.

The $Z^{\prime}$ widths into hadrons in the $\mathrm{U}(1)_{B}$ model, for $M_{Z^{\prime}}$ in the $3-3.7 \mathrm{GeV}$ range (or more precisely $M_{K^{0}}^{2} \ll M_{Z^{\prime}}^{2} / 4<M_{D^{0}}^{2}$ so that the decays are into mesons made up of $u, d, s$ quarks, and the phase space suppression can be neglected) are approximately given by

$$
\Gamma\left(Z_{B}^{\prime} \rightarrow \text { hadrons }\right) \approx \frac{g_{z}^{2}}{48 \pi} M_{Z^{\prime}},
$$

while for larger $M_{Z^{\prime}}$ the width increases by a factor of up to $4 / 3$ as decay channels involving $c$ quarks open up, and above $2 m_{b}$ by another factor of up to $5 / 4$. For illustration we use a benchmark set of values for the parameters:

$$
z_{\chi}=1 \text { or } 3, \frac{m_{\chi}}{M_{Z^{\prime}}}=\frac{1}{4} .
$$

The branching fractions of $Z^{\prime}$ into $\chi$ particles for this set of parameters, marked with an index 0 , are given in the $\mathrm{U}(1)_{B}$ model by

$$
\begin{aligned}
& B_{0}\left(Z_{B}^{\prime} \rightarrow \psi_{\chi} \bar{\psi}_{\chi}\right) \approx(42 \%, 87 \%) \text { for } z_{\chi}=(1,3), \\
& B_{0}\left(Z_{B}^{\prime} \rightarrow \phi_{\chi}^{\dagger} \phi_{\chi}\right) \approx(11 \%, 52 \%) \text { for } z_{\chi}=(1,3),
\end{aligned}
$$

for $M_{Z^{\prime}}$ in the $3-3.5 \mathrm{GeV}$ range, and by somewhat smaller values as $M_{Z^{\prime}}$ increases above $2 M_{D^{0}}$.

The $Z^{\prime}$ widths into hadrons in the $\mathrm{U}(1)_{d s}$ model, for $M_{Z^{\prime}} \gtrsim 3 \mathrm{GeV}$ (where the phase space suppression can be neglected for decays into mesons made up of $s$ or $d$ quarks) are given by

$$
\Gamma\left(Z_{d s}^{\prime} \rightarrow \text { hadrons }\right) \approx \frac{g_{z}^{2}}{16 \pi} M_{Z^{\prime}},
$$

The branching fractions of $Z^{\prime}$ into $\chi$ particles for the above set of parameters in the $\mathrm{U}(1) d s$ model are

$$
\begin{aligned}
& B_{0}\left(Z_{d s}^{\prime} \rightarrow \psi_{\chi} \bar{\psi}_{\chi}\right) \approx(25 \%, 75 \%) \text { for } z_{\chi}=(1,3), \\
& B_{0}\left(Z_{d s}^{\prime} \rightarrow \phi_{\chi}^{\dagger} \phi_{\chi}\right) \approx(5.1 \%, 33 \%) \text { for } z_{\chi}=(1,3) .
\end{aligned}
$$

for $M_{Z^{\prime}} \gtrsim 3 \mathrm{GeV}$, and decrease for smaller $M_{Z^{\prime}}$.

We now turn to deriving the constraints on the $Z^{\prime}$ in the $1-10 \mathrm{GeV}$ mass range, in the $\mathrm{U}(1)_{B}$ and $\mathrm{U}(1)_{d s}$ models. 


\subsection{Limits from monojet searches}

Hadron colliders set bounds on light $Z^{\prime}$ via mono-jet and mono-photon searches. For $M_{Z^{\prime}}<10 \mathrm{GeV}$ the strongest constraint comes from the CDF search $p \bar{p} \rightarrow j+\mathbb{E}_{T}$ [43], and is given by [30]:

$$
g_{z}\left[B\left(Z^{\prime} \rightarrow \chi \bar{\chi}\right)\right]^{1 / 2}< \begin{cases}0.12, & \text { for } \mathrm{U}(1)_{B}, \\ 0.11, & \text { for } \mathrm{U}(1)_{d s} .\end{cases}
$$

The limits from ATLAS $[44,45]$ and CMS $[46,47]$ are weaker due to stronger cuts imposed on missing energy and the jet $p_{T}$. In figure 1 we show the regions in the $\left(M_{Z^{\prime}}, g_{z}\right)$ plane excluded by these constraints for the benchmark values eq. (2.6).

\subsection{Invisibile quarkonium decays}

The searches for an invisible $\Upsilon$ decay constrain the $\mathrm{U}(1)_{B}$ model, while for the $\mathrm{U}(1)_{d s}$ model there is no such constraint since the $Z^{\prime}$ does not couple to $b$ quarks. The $Z_{B}^{\prime}$ exchange induces an $\Upsilon \rightarrow \chi \bar{\chi}$ decay. For a fermionic DM the ratio between the invisible decay and the decay into $\mu^{+} \mu^{-}$branching ratio is [29]:

$$
\frac{B\left(\Upsilon \rightarrow Z^{\prime} \rightarrow \text { invisible }\right)}{B\left(\Upsilon \rightarrow \gamma^{*} \rightarrow \mu^{+} \mu^{-}\right)}=\frac{g_{z}^{4} z_{\chi}^{2}}{16 g^{4} \sin ^{4} \theta_{W}}\left(\frac{M_{Z^{\prime}}^{2}}{M_{\Upsilon}^{2}}-1\right)^{-2}
$$

The most stringent bound on the $\Upsilon$ invisible branching fraction has been set by the BaBar Collaboration [48], $B(\Upsilon \rightarrow$ invisible $)<3 \times 10^{-4}$ at the $90 \%$ confidence level. This implies that the shaded region labelled " $\Upsilon$ " in figure 1 is excluded.

Similarly (except for the different electric charge of $c$ and $b$ quarks), a limit for $M_{Z^{\prime}}$ near $3 \mathrm{GeV}$ arises from $J / \psi$ decays, with the limit on invisible branching fraction given by $B(J / \psi \rightarrow$ invisible $)<7 \times 10^{-4}[49]$.

\subsection{Monophoton limits from BaBar data}

At tree level the $Z^{\prime}$ does not couple to leptons, but at one loop a kinetic mixing, $-\left(\epsilon_{B} / 2\right) Z_{\mu \nu}^{\prime} F^{\mu \nu}$, is generated. Therefore, bounds from dark photon searches apply also to a leptophobic $Z^{\prime}$. In the $1 \mathrm{GeV}<M_{Z^{\prime}}<10 \mathrm{GeV}$ mass range the strongest constraint comes from the BaBar monophoton search reinterpreted in terms of invisibly decaying $Z^{\prime}$ produced along with a single photon in $e^{+} e^{-}$collisions [50]. In the $\mathrm{U}(1)_{B}$ model the kinetic mixing at the BaBar center of mass energy $(E \sim 10 \mathrm{GeV})$ is [29]

$$
\epsilon_{B}(10 \mathrm{GeV}) \sim 10^{-2} g_{z}
$$

while for the $\mathrm{U}(1)_{d s}$ model the mixing is generated only below the strange quark mass and it is negligible. In figure 1 we present the bounds on $\gamma Z^{\prime}$ production taken from figure 5 of [50] and interpreted as a bound on $g_{z}$ using eq. (2.12).

\subsection{Anomaly cancellation versus collider limits on fermions}

The inclusion of a leptophobic gauge group $\mathrm{U}(1)_{z}$ requires new electrically-charged fermions which are vector-like with respect to the SM gauge group in order to cancel the gauge 

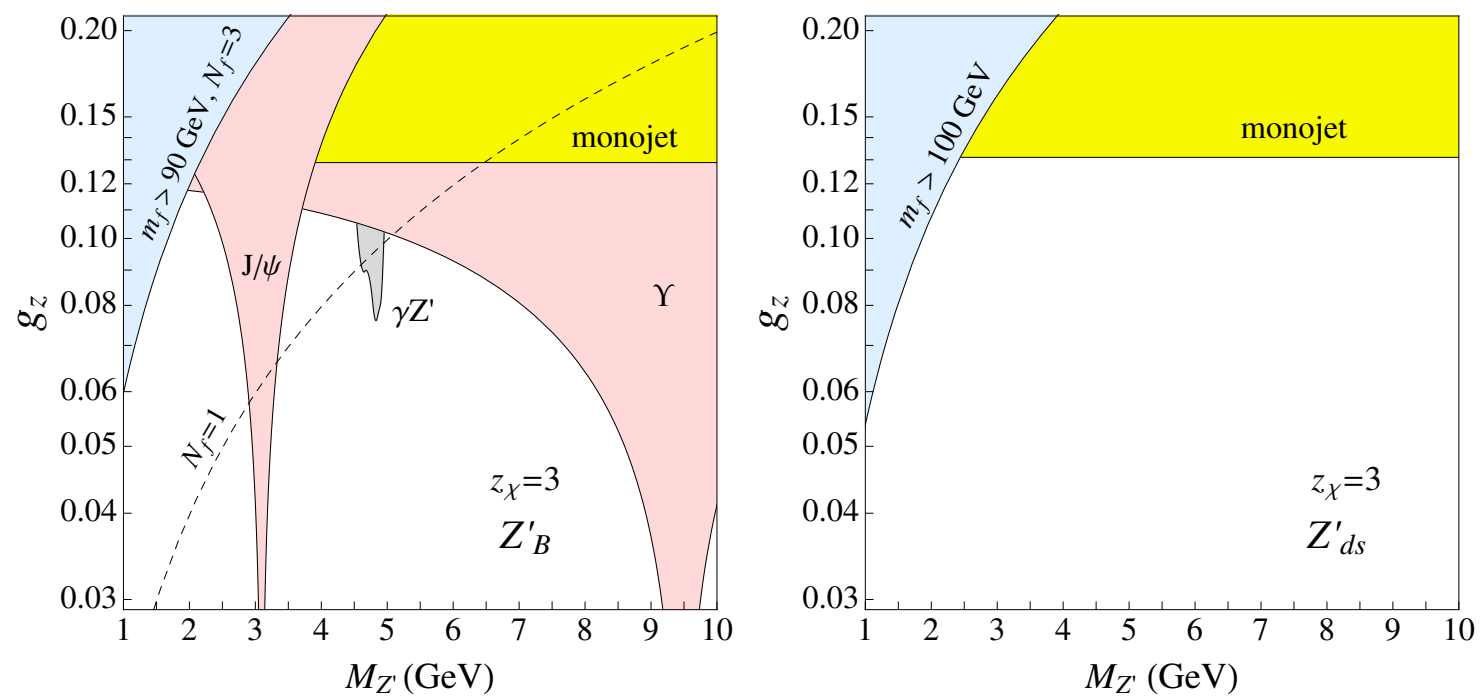

Figure 1. Constraints on the $\mathrm{U}(1)_{B}$ (left panel) and $\mathrm{U}(1)_{d s}$ (right panel) models from monojet collider searches (upper right-hand region), collider bounds on new fermions required to cancel gauge anomalies (upper left-hand corner), quarkonium decays (regions labelled by $J / \psi$ and $\Upsilon$ ), and the BaBar monophoton search (the $\gamma Z^{\prime}$ region).

anomalies. These fermions acquire a mass $m_{f}$ through a Yukawa coupling to a scalar $\varphi$ whose VEV breaks $\mathrm{U}(1)_{z}$. The collider limits on $m_{f}$ then translate then into an upper bound on the gauge coupling [3]:

$$
g_{z}=\frac{\sqrt{2} \lambda M_{Z^{\prime}}}{z_{\varphi} m_{f}} \lesssim 5.4 \times 10^{-2} \frac{1}{z_{\varphi}}\left(\frac{M_{Z^{\prime}}}{1 \mathrm{GeV}}\right)\left(\frac{100 \mathrm{GeV}}{m_{f}}\right)
$$

where $z_{\varphi}$ is the $\mathrm{U}(1)_{z}$ charge of $\varphi, \lambda$ is the Yukawa coupling, and we imposed a perturbativity bound $\lambda \lesssim 3.8$.

In the $\mathrm{U}(1)_{B}$ model, $z_{\varphi}=3$ if the minimal set of vectorlike fermions is included. If the charged fermions are almost degenerate with the neutral ones so that their collider signature involves only soft leptons, then they can be as light as $m_{f}=90 \mathrm{GeV}$, which is the LEP limit. If $N_{f}$ copies of the minimal set of vectorlike fermions are included, then $z_{\varphi}=3 / N_{f}$ (see [3] for a more detailed discussion). Large values of $N_{f}$ would increase the collider limit on $m_{f}$. The region excluded by eq. (2.13), shown in the left panel of figure 1 , is above the solid line labelled " $m_{f}>90 \mathrm{GeV}, N_{f}=3$ " in the case of three sets of vectorlike fermions, or above the dashed line labelled " $N_{f}=1$ " in the minimal $\mathrm{U}(1)_{B}$ model.

In the $\mathrm{U}(1)_{d s}$ model, $z_{\varphi}=1$, the LEP limit on $m_{f}$ is about $100 \mathrm{GeV}$, and there is less flexibility in changing the fermion content. The region excluded by eq. (2.13) in the right panel of figure 1 is above the line labelled " $m_{f}>100 \mathrm{GeV}$ ".

\section{Cosmological constraints on DM scenarios}

Let us outline some possible scenarios which give rise in our framework to a viable DM candidate. Since we are interested in DM of mass $m_{\chi}$ below a few $\mathrm{GeV}$, direct detection 
bounds are currently very mild [51-53]. The most stringent constraint is provided by the bounds on energy injection around redshifts $z \sim 100-1000$, coming from observations of the cosmic microwave background (CMB) [54-57]. This constrains the annihilation of DM into charged SM particles during recombination, and in particular rules out DM lighter than about $10 \mathrm{GeV}$ if it annihilates via $s$-wave processes. Therefore, CMB forces the dominant annihilation to be $p$-wave suppressed or to go into neutrinos. In our scenario a Dirac fermion $\psi_{\chi}$ annihilates into quarks via $s$-wave processes, and the thermal averaged cross section times velocity is [57]

$$
\left\langle\sigma\left(\psi_{\chi} \bar{\psi}_{\chi} \rightarrow q \bar{q}\right) v\right\rangle_{Z_{B}^{\prime}}=\frac{2}{9}\left\langle\sigma\left(\psi_{\chi} \bar{\psi}_{\chi} \rightarrow q \bar{q}\right) v\right\rangle_{Z_{d s}^{\prime}}=\frac{z_{\chi}^{2} g_{z}^{4} m_{\chi}^{2}}{48 \pi\left(M_{Z^{\prime}}^{2}-4 m_{\chi}^{2}\right)^{2}}
$$

Therefore, the CMB bound implies that $\psi_{\chi}$ can be a DM particle only if it is part of a hidden sector that is more complex than the minimal models of eq. (2.1). One possibility is to interpret the CMB bound as an upper limit on the $s$-wave annihilation into SM particles, that is [57]:

$$
\left\langle\sigma\left(\psi_{\chi} \bar{\psi}_{\chi} \rightarrow q \bar{q}\right) v\right\rangle \lesssim \frac{0.1 \mathrm{pb}}{f}\left(\frac{m_{\chi}}{1 \mathrm{GeV}}\right)
$$

where the ionizing efficiency factor is $f \approx 0.2$ for pions. Since the annihilation is suppressed, $\langle\sigma v\rangle<1 \mathrm{pb}$, the minimal model leads to overabundant DM, and therefore needs to be extended. A simple extension [14] includes a scalar $\eta$ that has a Yukawa coupling, $y_{1} \eta \bar{\psi}_{\chi} \psi_{\chi}$; if $m_{\eta}<m_{\chi}<M_{Z^{\prime}} / 2$ the annihilation $\psi_{\chi} \bar{\psi}_{\chi} \rightarrow 2 \eta$ dominates, and gives the correct relic abundance, e.g. for, $y_{1} \sim 0.05, m_{\chi}=1 \mathrm{GeV}$ and $m_{\eta} \lesssim O(0.1) \mathrm{GeV}$. This annihilation mode is $p$-wave suppressed and therefore CMB safe. The $\eta$ scalar can then decay into SM particles via a small Higgs portal coupling. The condition in eq. (3.2) is satisfied for values of $g_{z}$ below the dashed red curves in figure 2. Note that indirect DM searches could impose some constraints on this scenario in the case of $\psi_{\chi} \bar{\psi}_{\chi} \rightarrow \eta \eta \rightarrow e^{+} e^{-} e^{+} e^{-}, 4 \gamma$ annihilations.

Another way to satisfy the CMB constraint is to suppress the $\psi_{\chi}$ relic density rather than the annihilation cross section into SM charged particles. This possibility requires a substantially larger cross section than the one corresponding to the correct relic abundance [57]:

$$
\langle\sigma(\psi \bar{\psi} \rightarrow q \bar{q}) v\rangle \gtrsim 16 \mathrm{pb} .
$$

This is self-consistent if $\psi_{\chi}$ is a subdominant DM component. Alternatively the minimal model can be part of an asymmetric DM model [58], so that eq. (3.3) represents the condition of depletion of the symmetric component [57]. Indeed in models of asymmetric DM, annihilation during recombination can be easily suppressed. The region above the solid blue lines in figure 2 satisfies eq. (3.3) for $m_{\chi}=M_{Z^{\prime}} / 4$. Both an underabundant and an asymmetric DM population would suppress signals for indirect DM searches.

The shaded region in figure 2, between the two lines corresponding to the two scenarios just described, is not necessarily ruled out: if both ingredients discussed above are present then the CMB constraints can be accommodated. We see that the CMB constraints are very model dependent, and hence there is still a large region of the parameter space yet unexplored which leads to viable DM scenarios where Dirac fermions interact with SM quarks via $s$-wave processes. 

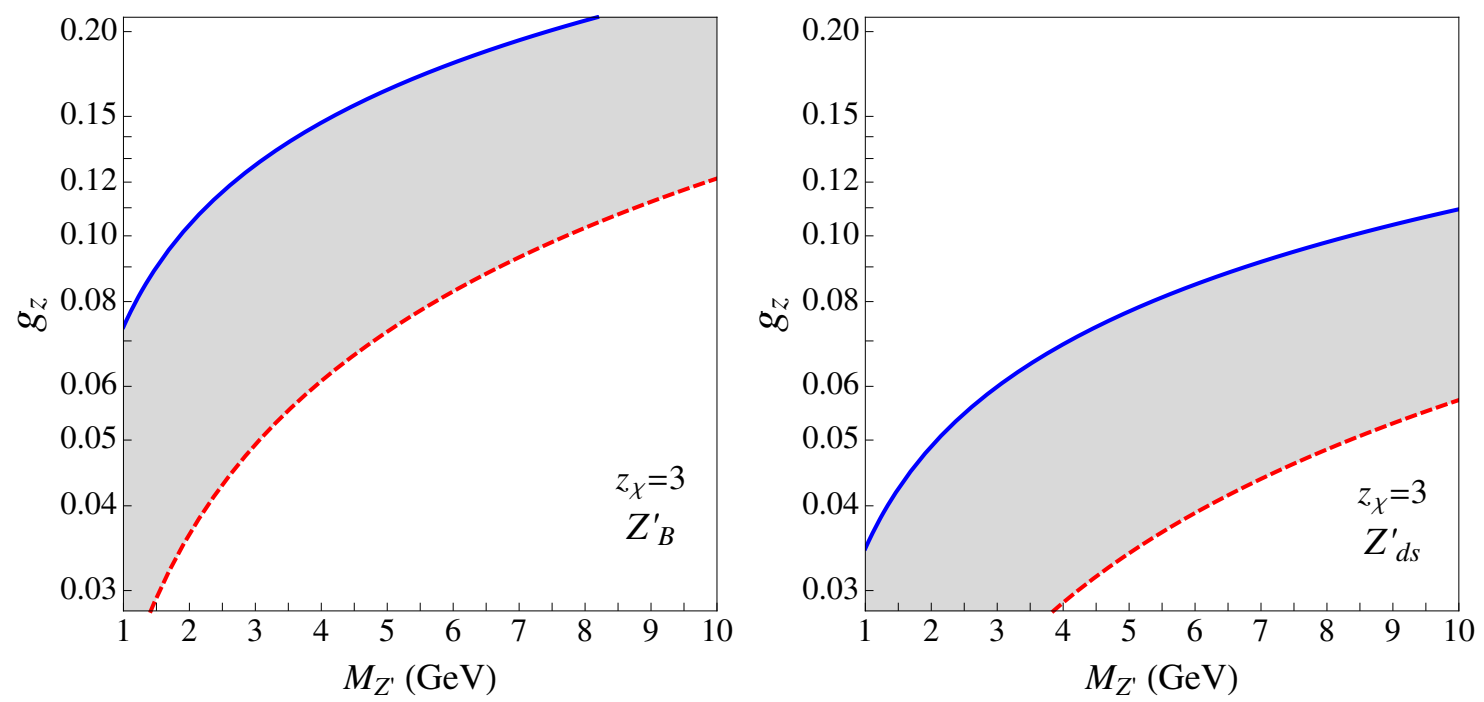

Figure 2. $\mathrm{CMB}$ constraints for fermonic $\mathrm{DM} \psi_{\chi}$ in the $\mathrm{U}(1)_{B}$ (left panel) and $\mathrm{U}(1)_{d s}$ (right panel) models. The region above the solid (blue) line is viable if $\psi_{\chi}$ is asymmetric DM or a subdominant DM component. The region below the dashed (red) line is also CMB safe since the $s$-wave annihilation into quarks is small; the correct relic abundance is obtained for example via $p$-wave annihilation into new light scalars. The shaded region requires a more complex hidden sector (asymmetric DM with the symmetric component depleted by annihilation into new states).

In the case of scalar DM, $\phi_{\chi}$, the annihilation cross section into quarks is [59]:

$$
\left\langle\sigma\left(\phi_{\chi} \phi_{\chi}^{*} \rightarrow q \bar{q}\right) v\right\rangle_{Z_{B}^{\prime}}=\frac{2}{9}\left\langle\sigma\left(\phi_{\chi} \phi_{\chi}^{*} \rightarrow q \bar{q}\right) v\right\rangle_{Z_{d s}^{\prime}}=\frac{v^{2} z_{\chi}^{2} g_{z}^{4} m_{\chi}^{2}}{288 \pi\left(M_{Z^{\prime}}^{2}-4 m_{\chi}^{2}\right)^{2}},
$$

where $v \sim 0.3$ is the DM velocity at freeze out. This is $p$-wave suppressed, and hence CMB safe. Limits from DM indirect searches in this case could be stronger than the CMB ones (see [60] where the focus is on DM annihilating into electrons). However, these bounds are not strong enough to rule out a thermal DM particle heavier than $\sim 100 \mathrm{MeV}$. Relatively large values of the gauge coupling are typically needed in order to achieve the correct relic abundance (which requires $\langle\sigma v\rangle \sim 1.5 \mathrm{pb}$ at freeze out for light DM [61]). For $m_{\chi} \approx M_{Z^{\prime}} / 4$ and $z_{\chi}=3$ the correct relic abundance is obtained with $g_{z} \sim 0.06\left(M_{Z^{\prime}} / 1 \mathrm{GeV}\right)^{1 / 2}$ for the $\mathrm{U}(1)_{B}$ model and $g_{z} \sim 0.04\left(M_{Z^{\prime}} / 1 \mathrm{GeV}\right)^{1 / 2}$ for the $\mathrm{U}(1)_{d s}$ model. Since the present bounds on scalar DM are similar to the ones for fermion DM presented in figure 1, we conclude that there are still open regions of the parameter space where the minimal models of eq. (2.1) give a scalar thermal DM candidate.

\section{DM production through proton scattering off nucleons}

Having examined the bounds on $\mathrm{GeV}$-scale leptophobic $Z^{\prime}$ bosons decaying into DM particles, we now proceed to discuss the potential sensitivity of proton fixed target experiments to this scenario.

We assume that the $Z^{\prime}$ boson is produced on-shell and then decays into DM particles $\left(M_{Z^{\prime}}>2 m_{\chi}\right)$. This way the DM particles are produced resonantly: $p N \rightarrow Z^{\prime} \rightarrow \chi \bar{\chi}$, where 
$N$ indicates the nucleon inside the target. The cross section for proton-nucleon scattering, computed within the parton model, is

$$
\sigma(p N \rightarrow \chi \bar{\chi})=\int d x_{1} d x_{2} \sum_{q} f_{q \mid N}\left(x_{1}\right) f_{\bar{q} \mid N}\left(x_{2}\right) \hat{\sigma}_{q}\left(x_{1} x_{2} s\right) B\left(Z^{\prime} \rightarrow \chi \bar{\chi}\right)
$$

where $B\left(Z^{\prime} \rightarrow \chi \bar{\chi}\right)$ is the branching fraction of the $Z^{\prime}$ boson into DM particles. If the vector boson is produced on-shell, the tree-level partonic cross section is

$$
\hat{\sigma}_{q}(\hat{s})=\frac{g_{z}^{2}}{3}\left(z_{q_{L}}^{2}+z_{q_{R}}^{2}\right) \delta\left(\hat{s}-M_{Z^{\prime}}^{2}\right) .
$$

In the $\mathrm{U}(1)_{B}$ model $z_{q_{L}}=z_{q_{R}}=1 / 3$, so that the proton and the neutron cross sections are the same. In the $\mathrm{U}(1)_{d s}$ model only the right-handed $d$ and $s$ quarks have nonzero charges $\left(z_{q_{R}}^{2}=1\right.$ for $\left.q=d, s\right)$, leading to different proton-neutron and proton-proton cross sections: $\sigma\left(p n \rightarrow Z_{d s}^{\prime}\right) \simeq 2 \sigma\left(p p \rightarrow Z_{d s}^{\prime}\right)$. As a result, the average proton-nucleon cross section is material dependent. For a target of atomic mass $A_{\mathrm{T}}$ and atomic number $Z_{\mathrm{T}}$, the average $p N$ cross section is

$$
\sigma(p N \rightarrow \chi \bar{\chi})_{\mathrm{T}} \simeq \frac{1}{A_{\mathrm{T}}}\left(Z_{\mathrm{T}} \sigma(p p \rightarrow \chi \bar{\chi})+\left(A_{\mathrm{T}}-Z_{\mathrm{T}}\right) \sigma(p n \rightarrow \chi \bar{\chi})\right) .
$$

Comparing the $Z^{\prime}$ production rate with the total proton-proton cross section, $\sigma(p p)$, which for a $120 \mathrm{GeV}$ beam is given by $\sigma(p p) \approx 40 \mathrm{mb}$ (see figure 46.10 [1]), we find the number of DM particles produced in the target:

$$
N_{\chi}^{\mathrm{T}}=\frac{2 N_{\mathrm{POT}}}{\sigma(p p)} \sigma(p N \rightarrow \chi \bar{\chi})_{\mathrm{T}}
$$

where $N_{\text {POT }}$ is the number of protons on target.

Using MadGraph 5 [62] to compute the production cross section, and FeynRules [63] to implement the $Z^{\prime}$ models, we find the number $N_{\chi}^{\mathrm{T}}$ of produced DM particles shown in figure 3 for $10^{21}$ protons on target. We focus on $M_{Z^{\prime}}>2 \mathrm{GeV}$ because the validity of the parton model is questionable in the case of lighter $Z^{\prime}$ production. The $Z_{d s}^{\prime}$ line shown in figure 3 corresponds to an isospin-symmetric target $\left(A_{T}=2 Z_{T}\right)$. More generally, the number of DM particles produced has only a mild dependence on $Z_{T} / A_{T}$ (and is material independent in the $Z_{B}^{\prime}$ model).

The value of the gauge coupling used in figure 3 is $g_{z}=0.1$; for other values, $N_{\chi}$ scales as $\left(g_{z} / 0.1\right)^{2}$. The branching fraction for $Z^{\prime} \rightarrow \chi \bar{\chi}$ used in figure 3 is $100 \%$; more realistic choices, discussed in section 2 , depend on $m_{\chi}, z_{\chi}$ and on whether $\chi$ is a fermion or a scalar (in the case where $\chi$ is a Dirac fermion and $z_{\chi}=3$, the branching fraction is large, of about $87 \%$ for $Z_{B}^{\prime}$ and $75 \%$ for $Z_{d s}^{\prime}$ ).

\section{DM flux through detectors}

We now proceed to compute how many of the produced dark matter particles pass through detectors, as well as their energy distribution. We will discuss both off-axis and on-axis detectors, with examples given by the $\mathrm{NO} \nu \mathrm{A}$ and MINOS near detectors. 


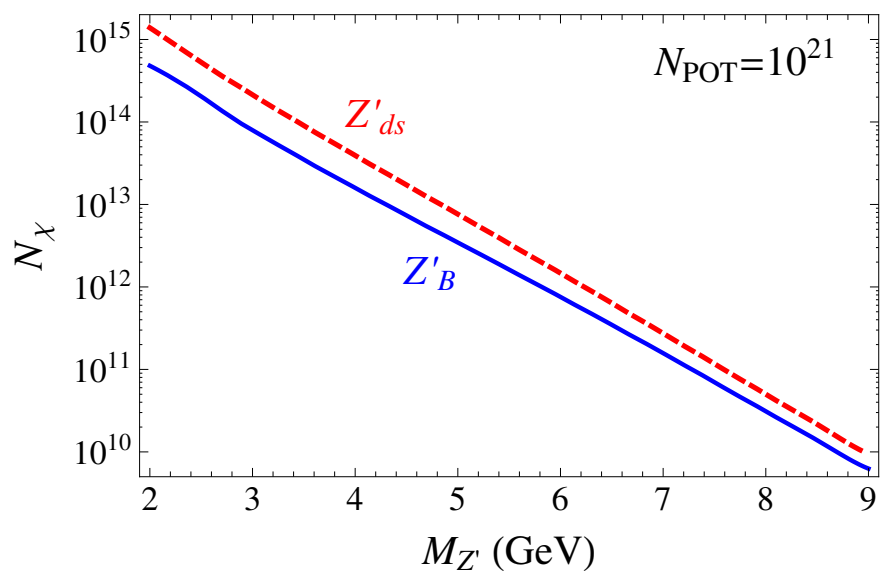

Figure 3. Number of $\chi$ or $\bar{\chi} \mathrm{DM}$ particles produced for $N_{\mathrm{POT}}=10^{21}$ protons of $120 \mathrm{GeV}$ scattering off a fixed target which is thick enough to stop all incoming protons. The two lines are predicted in the $\mathrm{U}(1)_{B}$ (solid blue line) and $\mathrm{U}(1)_{d s}$ models (dashed red line, for $A_{T}=2 Z_{T}$ ) with $g_{z}=0.1$. The $Z^{\prime} \rightarrow \chi \bar{\chi}$ branching fraction used here is $100 \%$, corresponding to $z_{\chi} \gg 1$; for smaller $z_{\chi}$ the branching fraction depends on $M_{Z}^{\prime}, m_{\chi}$ and the $\chi$ spin (see section 2).

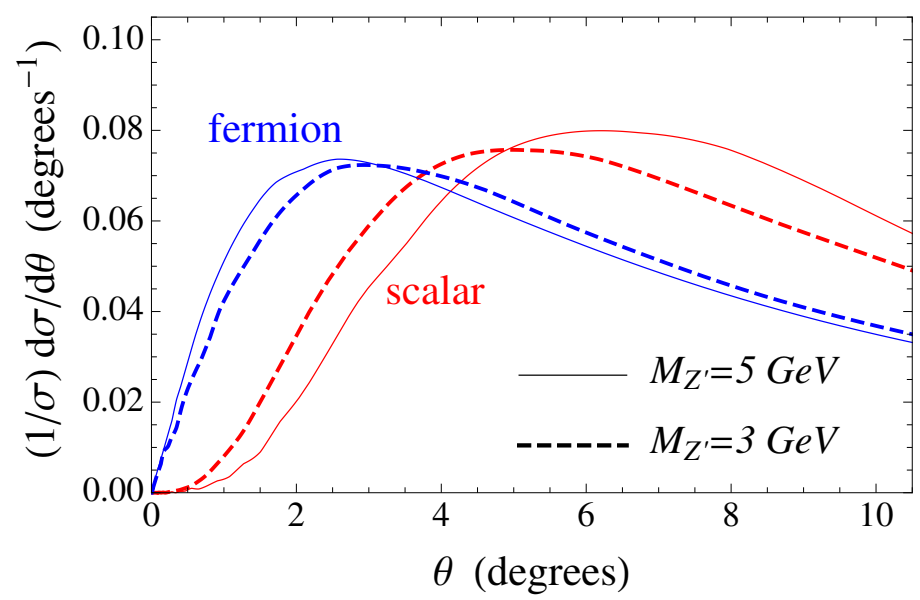

Figure 4. Polar angle distribution in the lab frame of the DM particles produced in the $p N \rightarrow$ $Z^{\prime} \rightarrow \chi \bar{\chi}$ process, for $M_{Z^{\prime}}=3 \mathrm{GeV}$ (dashed lines) or $M_{Z^{\prime}}=5 \mathrm{GeV}$ (solid lines), when $\chi$ is a Dirac fermion or a complex scalar.

\subsection{Angular distribution of DM particles}

Let us denote the polar angle in the lab frame (i.e., the angle between the direction of one of the DM particles produced in $Z^{\prime}$ decays and the beam direction) by $\theta$. Using the output of the MadGraph simulation, we obtain the polar angular distributions shown in figure 4 in the cases where the DM particle is a Dirac fermion or a complex scalar. The fermion angular distribution is more spread and peaked towards smaller angles than the scalar one. This different behavior can be understood by considering the scalar and fermion distributions in the center-of-mass frame, $d \sigma / d \theta \propto\left(1 \mp \cos ^{2} \theta\right) \sin \theta$, and then boosting to the lab frame. Note that our choice of vector coupling of $Z^{\prime}$ to the fermion DM implies that the angular distribution is the same independently of the $Z^{\prime}$ couplings to quarks. 


\begin{tabular}{|c|c|c|ccc|cc|}
\hline Detector & DM source & distance & $\theta_{\min }$ & $\theta_{\max }$ & $\phi_{\max }$ & $\epsilon_{\text {det }}($ fermion $)$ & $\epsilon_{\text {det }}$ (scalar $)$ \\
\hline MINOS & absorber & $270 \mathrm{~m}$ & 0 & $0.48^{\circ}$ & $180^{\circ}$ & $6 \times 10^{-3}$ & $10^{-4}$ \\
MINOS & target & $950 \mathrm{~m}$ & 0 & $0.19^{\circ}$ & $180^{\circ}$ & $8 \times 10^{-4}$ & $3 \times 10^{-6}$ \\
\hline NO $\nu \mathrm{A}$ & absorber & $240 \mathrm{~m}$ & $2.6^{\circ}$ & $3.6^{\circ}$ & $18^{\circ}$ & $3 \times 10^{-3}$ & $2 \times 10^{-3}$ \\
$\mathrm{NO} \nu \mathrm{A}$ & target & $920 \mathrm{~m}$ & $0.68^{\circ}$ & $0.93^{\circ}$ & $18^{\circ}$ & $4 \times 10^{-4}$ & $3 \times 10^{-5}$ \\
\hline
\end{tabular}

Table 1. Geometrical parameters for particles produced in the absorber or the target and passing through the MINOS or NO $\nu \mathrm{A}$ near detectors. The polar angle satisfies $\theta_{\min }<\theta<\theta_{\max }$, while the azimuthal angle satisfies $0 \leq \phi \leq \phi_{\max }$. The geometric acceptance of the detector $\epsilon_{\operatorname{det}}$ (shown here for $M_{Z^{\prime}}=3 \mathrm{GeV}$ ) depends on the DM spin.

The Carbon target used in the NuMi beam line stops about $85 \%$ of the incoming protons, while the remaining $15 \%$ of protons travel through the $675 \mathrm{~m}$ long vacuum pipe and hit the absorber, which is mostly made of iron. These two sets of protons give comparable contributions to the total number of DM particles inside the MINOS and $\mathrm{NO} \nu \mathrm{A}$ near detectors. The smaller number of protons reaching the absorber is compensated by the larger coverage of the detector along the azimuthal angle $\phi$. In table I we list the approximate angular cuts required for a DM particle produced in the absorber or the target to reach the MINOS or $\mathrm{NO} \nu \mathrm{A}$ near detectors.

We compute the geometrical acceptance of the detector, $\epsilon_{\text {det }}$, by imposing angular cuts on the DM particles produced in the simulated events. In the case of a Dirac fermion, we find $\epsilon_{\operatorname{det}} \gtrsim O\left(10^{-3}\right)$ both for MINOS and $\mathrm{NO} \nu \mathrm{A}$ near detectors for particles produced at the absorber, while for particles produced at the target the acceptance is smaller by an order of magnitude due to the larger distance. The values of the acceptance are given in table I for $M_{Z^{\prime}}=3 \mathrm{GeV}$. For a scalar, $d \sigma / d \theta$ vanishes faster for $\theta \rightarrow 0$, so that the acceptance of on-axis detectors is suppressed. Therefore, this offers a possibility to measure the spin of a discovered DM particle via a parallel MINOS and $\mathrm{NO} \nu \mathrm{A}$ analysis.

Higher-order processes that include real radiation, $p p \rightarrow Z^{\prime}+$ jets, can potentially change the scalar angular distribution. As a crude approximation, we computed the tree-level production of $Z^{\prime}$ together with one or two hard jets, imposing a jet- $p_{t}$ cut of $1 \mathrm{GeV}$, and we found that these processes are not large enough compared to $p p \rightarrow Z^{\prime}$ to change qualitatively the above result.

\subsection{Energy distribution of DM particles}

The energy distributions of DM particles that enter the $\mathrm{NO} \nu \mathrm{A}$ and MINOS near detectors are shown in figure 5 for $M_{Z^{\prime}}=3 \mathrm{GeV}$. The DM particles inside the detectors are fairly energetic compared to the neutrinos (produced mostly in pion decays), especially for $\mathrm{NO} \nu \mathrm{A}$. The neutrinos enter the $\mathrm{NO} \nu \mathrm{A}$ near detector with a peak energy around $3 \mathrm{GeV}$; a tail of high-energy neutrinos $\left(E_{\nu} \gtrsim 10 \mathrm{GeV}\right)$ is produced mostly by kaon and heavier meson decays. The difference in the energy profile between DM and neutrino can be used to reduce 


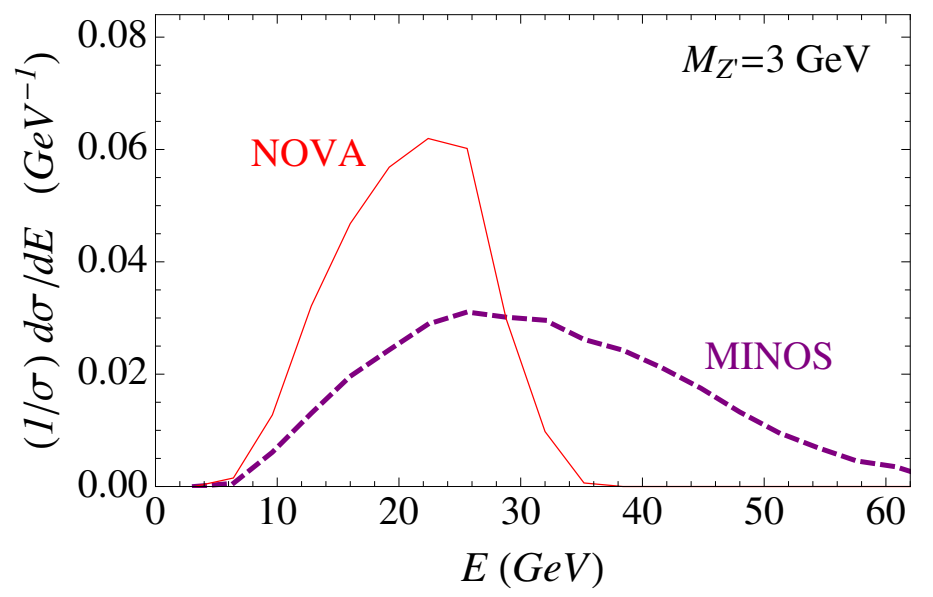

Figure 5. Energy distribution of fermonic DM particles produced in the absorber and passing through the $\mathrm{NO} \nu \mathrm{A}$ or MINOS near detectors for $M_{Z^{\prime}}=3 \mathrm{GeV}$.

the neutrino background. This can be done considering processes where DM transfer a significant energy to nucleus, and imposing a cut on the energy of the hadronic activity.

The difference between energy distributions of DM and neutrinos is less pronounced for on-axis detectors such as MINOS, where the neutrinos have a wider energy distribution with a long tail $[8,9,64]$. However, a dedicated search for MINOS near detector would also be possible and highly interesting, particularly for reasons mentioned in section 5.1 related to the distinction between DM beams of scalars or fermions.

\section{$6 \quad$ DM scattering inside the detector}

DM particles may scatter off the nucleons in the detector by exchanging a $Z^{\prime}$ boson in the $t$ channel, and producing neutral-current events. Let us study the interaction between the DM particles and nucleons inside the detector. The cross section for DM interacting with nucleons can be much larger than the neutrino one since it is mediated by a lighter boson. For momentum transfers much smaller than $M_{Z^{\prime}}$, the ratio of the DM to neutrino cross sections for neutral-current events is

$$
\begin{aligned}
\frac{\sigma(\chi N \rightarrow \chi j)}{\sigma(\nu N \rightarrow \nu j)} & \approx C_{N}\left(Z^{\prime}\right) \frac{z_{\chi}^{2}}{4}\left(\frac{g_{z}}{g} \cos \theta_{W} \frac{M_{Z}}{M_{Z^{\prime}}}\right)^{4} \\
& \approx 23 C_{N}\left(Z^{\prime}\right) z_{\chi}^{2}\left(\frac{g_{z}}{0.1}\right)^{4}\left(\frac{4 \mathrm{GeV}}{M_{Z^{\prime}}}\right)^{4},
\end{aligned}
$$

where $j$ stands for any hadronic final state. $C_{N}\left(Z^{\prime}\right)$ is a coefficient of order one that depends on whether the nucleon $N$ is a proton $(N=p)$ or neutron $(N=n)$, as well as on the $Z^{\prime}$ model; neglecting the interactions of sea quarks and nuclear form factors, this coefficient takes the values

$$
C_{p}\left(Z_{B}^{\prime}\right) \approx \frac{2}{3}\left(\frac{3}{4}-\frac{5}{3} s_{W}^{2}+2 s_{W}^{4}\right)^{-1} \approx 1.42, \quad C_{p}\left(Z_{d s}^{\prime}\right) \approx \frac{3}{2} C_{p}\left(Z_{B}^{\prime}\right)
$$




$$
C_{n}\left(Z_{B}^{\prime}\right) \approx \frac{2}{3}\left(\frac{3}{4}-\frac{4}{3} s_{W}^{2}+\frac{16}{9} s_{W}^{4}\right)^{-1} \approx 1.25, \quad C_{n}\left(Z_{d s}^{\prime}\right) \approx 3 C_{n}\left(Z_{B}^{\prime}\right),
$$

where $s_{W} \equiv \sin \theta_{W}$ is evaluated at a scale of a few $\mathrm{GeV}\left(\sin ^{2} \theta_{W} \approx 0.235\right)$. For a DM charge under the new $\mathrm{U}(1)$ group of $z_{\chi}=3$, the values of the $Z^{\prime}$ gauge coupling and mass shown in figure 1 allow the ratio in eq. (6.1) to be as large as $10^{3}$. However, even with such a large cross section for DM-nucleon scattering, the total number of DM events in the detector is much smaller than that of neutrino neutral-current events, because of the very large QCD production of pions and other mesons, whose decays generate the neutrino beam.

Before discussing selection cuts that reduce the neutrino background, let us compute the total number of DM events in the detector. The average DM-nucleon scattering cross section in the detector can be written as

$$
\sigma(\chi N \rightarrow \chi j)_{\mathrm{d}}=\frac{1}{A_{\mathrm{d}}}\left(Z_{\mathrm{d}} \sigma(\chi p)+\left(A_{\mathrm{d}}-Z_{\mathrm{d}}\right) \sigma(\chi n)\right),
$$

where $j$ stands for any set of hadrons. The DM-proton and DM-neutron cross sections, $\sigma(\chi p)$ and $\sigma(\chi n)$, are functions of the incoming DM energy $E_{\chi}$. The number of DM particles which are produced in the target and enter the detector is $f_{\mathrm{T}} \epsilon_{\operatorname{det}}^{\mathrm{T}} N_{\chi}$, where $f_{\mathrm{T}}$ is the fraction of incoming protons stopped in the target $\left(f_{\mathrm{T}} \approx 0.85\right.$ for the NuMI beam line), $N_{\chi}$ is shown in figure 3, and the geometric acceptance $\epsilon_{\text {det }}^{\mathrm{T}}$ is given in table I. Multiplying this number of particles by the fraction of those that have energy between $E_{\chi}$ and $E_{\chi}+d E_{\chi}$ (shown in figure 5) gives

$$
d N_{\mathrm{T}}\left(E_{\chi}\right)=f_{\mathrm{T}} \epsilon_{\mathrm{det}}^{\mathrm{T}} N_{\chi}\left(\frac{1}{\sigma} \frac{d \sigma}{d E_{\chi}}\right)(p N \rightarrow \chi \bar{\chi})_{\mathrm{T}} d E_{\chi} .
$$

For a detector of density $\rho_{d}$ and length $L_{d}$, the number of signal events due to the beam produced in the target is given by

$$
S_{\mathrm{T}}=\frac{L_{d} \rho_{d}}{m_{p}} \int d N_{\mathrm{T}}\left(E_{\chi}\right) \sigma(\chi N \rightarrow \chi j)_{\mathrm{d}} .
$$

For the $\mathrm{NO} \nu \mathrm{A}$ near detector $\rho_{d} \approx 1263 \mathrm{~kg} / \mathrm{m}^{3}$ and $L_{d} \approx 14.3 \mathrm{~m}$, while for the MINOS near detector $\rho_{d} \approx 3237 \mathrm{~kg} / \mathrm{m}^{3}$ and $L_{d} \approx 16.6 \mathrm{~m} ; A_{d} \approx 2 Z_{d}$ is a good approximation for both detectors.

An expression analogous to eq. (6.5) can be obtained for the number $\left(S_{\mathrm{A}}\right)$ of signal events due to the beam produced in the absorber, by replacing the quantities carrying a $\mathrm{T}$ index with the ones corresponding to the absorber (marked by an A index). Given that all incoming protons are stopped in the absorber, $f_{\mathrm{A}}=1-f_{\mathrm{T}}$. The total number of signal events is thus $S_{\mathrm{T}}+S_{\mathrm{A}}$.

Without imposing cuts there are $O\left(10^{7}\right)$ neutral-current neutrino scattering events, which is a too large background for allowing sensitivity to our signal. Thus, it is necessary to find some selection cuts that reduce the neutrino background without reducing the DM signal too much. If we label the incoming and outgoing $\chi$ four-momenta by $k^{\mu}$ and $k^{\prime \mu}$ respectively, and the momentum transfer by $q^{\mu}=k^{\mu}-k^{\prime \mu}$, then the DIS regime is realized for $Q^{2}>m_{p}^{2}$. For lighter mediators, $M_{Z^{\prime}}<1 \mathrm{GeV}$, the most relevant region for fixed target 

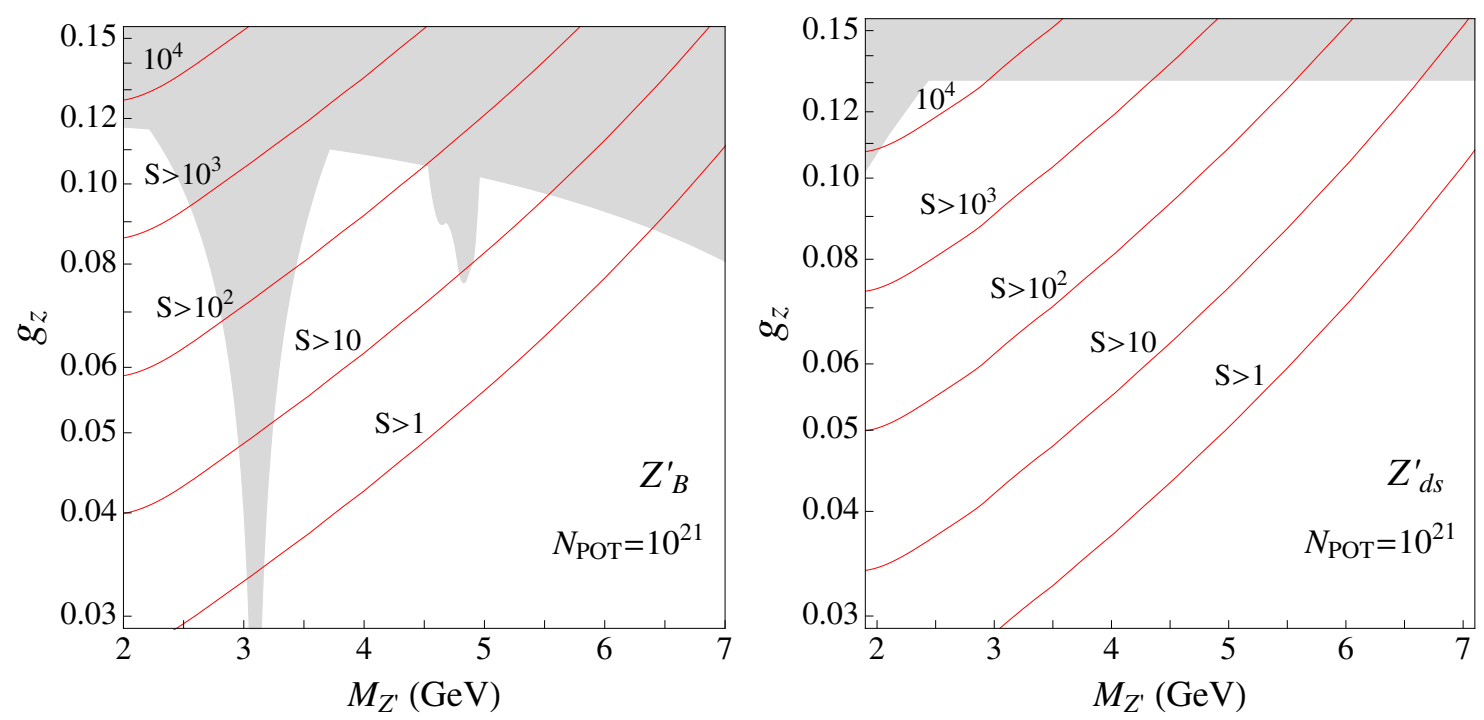

Figure 6. Predicted number of DM scattering events with hadronic energy above $2 \mathrm{GeV}$ in the $\mathrm{NO} \nu \mathrm{A}$ near detector, shown as red contour lines, for the $\mathrm{U}(1)_{B}$ (left) and $\mathrm{U}(1)_{d s}$ (right) models with $z_{\chi}=3$. The shaded regions are excluded by other experiments (see figure 1 ).

experiments is $Q^{2}=-q^{2}<1 \mathrm{GeV}[65]$. This is explained by the $Q^{2}$ dependence of the cross-section: $1 /\left(M_{Z^{\prime}}^{2}+Q^{2}\right)$. For heavier $Z^{\prime}$ bosons, of mass around a few $\mathrm{GeV}$, we expect the DIS regime to dominate. Consequently, we expect that it is helpful to impose a cut on the energy $E_{j}$ of the hadronic activity produced by the DM particle in the detector.

The peak energy of the neutrinos that enter the $\mathrm{NO} \nu \mathrm{A}$ detector is near $3 \mathrm{GeV}$, while the energy hadronic activity due to the neutrinos peaks at smaller values. We impose a cut $E_{j}>2 \mathrm{GeV}$, as stronger cuts reduce the signal too much in some cases. We expect this cut to not be sufficient to reduce enough the huge neutrino background. Therefore, additional strategies may be required, such as timing the delay of DM, or running in the proton beam-dump mode [11-15].

We computed the $\sigma(\chi N \rightarrow \chi j)_{\mathrm{d}}$ cross section after this cut with MadGraph 5; in figure 6 we show the regions in the $\left(M_{Z^{\prime}}, g_{z}\right)$ plane (above the red lines) where the number of $\mathrm{DM}$ scattering events in the $\mathrm{NO} \nu \mathrm{A}$ detector satisfies $S>10^{n}, n=1, \ldots, 4$, for $z_{\chi}=3$. We also show there the regions (shaded) excluded by various experiments discussed in section 2. We find that the number of signal events $S$ can be as large as $10^{3}$ for $Z_{B}^{\prime}$ and $10^{4}$ for $Z_{d s}^{\prime}$, with $N_{\mathrm{POT}}=10^{21}$. It appears that $\mathrm{NO} \nu \mathrm{A}$ may be able to explore a viable region of parameter space.

The MINOS near detector may also be able to probe the case of fermionic $\chi$, even though the background is larger because the neutrinos traveling closer to the axis are typically more energetic [64]. Therefore, we urge both the $\mathrm{NO} \nu \mathrm{A}$ and MINOS Collaborations to perform dedicate analyses to probe the presence of a DM beam. 


\section{Outlook}

It is important for DM searches to be as broad as possible in order to cover the wide range of allowed masses and the many potential portals to the visible sector. Proton fixed target experiments offer the possibility to explore a region of the parameter space left unconstrained by the existing searches involving direct detection, collider searches, or invisible quarkonium decays. The high beam intensity of these experiments makes them a promising ground for testing models with light DM particles.

In this paper we have studied the potential sensitivity of the neutrino near detectors to a DM beam produced at the Fermilab Main Injector, in the NuMI beam line. We have considered DM candidates, either a fermion or a scalar, charged under a new leptophobic gauge group, with the associated $Z^{\prime}$ boson having a mass $M_{Z}^{\prime}$ in the $1-10 \mathrm{GeV}$ range. Assuming that the DM mass satisfies $m_{\chi}<M_{Z^{\prime}} / 2$, pairs of DM particles may be resonantly produced when the $120 \mathrm{GeV}$ proton beam scatters off the target or is dumped in the absorber.

We have found a potentially interesting reach for these experiments provided that an efficient way to reduce the neutrino background is found. We have outlined possible solutions in this sense, focusing on the $\mathrm{NO} \nu \mathrm{A}$ near detector since its off-axis position is better suited than the MINOS one for reducing the neutrino background.

Even though the $\mathrm{NO} \nu \mathrm{A}$ near detector is better positioned for our purpose, the MINOS near detector can also be sensitive to a DM beam, especially in the case of fermionic DM particles. If a DM particle is discovered, then a parallel analysis done by the MINOS and $\mathrm{NO} \nu \mathrm{A}$ collaborations might reveal its spin. The Miner $\nu$ a detector is on-axis, but about $10 \%$ closer to the absorber than MINOS, so it may provide further tests.

The neutrino detectors along the Booster beam line at Fermilab may also be used to probe the presence of DM beams generated at the NuMI beam line. The MiniBoone detector is $6.3^{\circ}$ off-axis when viewed from the NuMI target [66], and at more than $90^{\circ}$ off-axis when viewed from the NuMI absorber; it also happens to be closer to the absorber by a factor of 2 compared to the MINOS near detector. The MicroBoone detector [67] is $\sim 8^{\circ}$ off-axis when viewed from the NuMI target, and the proposed LAr1-ND detector [68] would be $\sim 30^{\circ}$ off-axis.

The proposed LBNF [69] beam line at Fermilab would have a substantially larger $N_{\text {POT }}$ than the NuMI beam line. Thus, if a near detector is built close enough to the LBNF target or absorber, then the expected number of DM events can be an order of magnitude higher than in $\mathrm{NO} \nu \mathrm{A}$. Furthermore, the steep downwards slope of the proposed LBNF beam, in conjuction with the shape of the DM beam (a conic shell originating at the target and another one originating at the absorber), offers interesting physics opportunities if two or more near detectors are placed off-axis.

\section{Acknowledgments}

We thank Prateek Agrawal, Brian Batell, Pilar Coloma, Patrick Fox, Martin Frank, Lisa Goodenough, Roni Harnik, Lauren Hsu, Siva Kasetti, Ian Lewis, Tongyan Lin, David McKeen, Denis Perevalov, Robert Plunkett, Adam Ritz and David Schmitz for valuable 
discussions and comments. Fermilab is operated by Fermi Research Alliance, LLC under Contract No. DE-AC02-07CH11359 with the United States Department of Energy.

Open Access. This article is distributed under the terms of the Creative Commons Attribution License (CC-BY 4.0), which permits any use, distribution and reproduction in any medium, provided the original author(s) and source are credited.

\section{References}

[1] Particle Data Group collaboration, K. Olive et al., Review of particle physics, Chin. Phys. C 38 (2014) 090001 [INSPIRE].

[2] P. Cushman et al., Working group report: WIMP dark matter direct detection, arXiv: 1310.8327 [INSPIRE].

[3] B.A. Dobrescu and C. Frugiuele, Hidden GeV-scale interactions of quarks, Phys. Rev. Lett. 113 (2014) 061801 [arXiv: 1404.3947] [INSPIRE].

[4] S. Tulin, New weakly-coupled forces hidden in low-energy QCD, Phys. Rev. D 89 (2014) 114008 [arXiv: 1404.4370] [INSPIRE].

[5] K. Anderson et al., The NuMI facility technical design report, FERMILAB-DESIGN-1998-01, Fermilab, Batavia U.S.A. (1998) [inSPIRE].

[6] NOvA collaboration, R.B. Patterson, The NOvA experiment: status and outlook, Nucl. Phys. Proc. Suppl. B 235-236 (2013) 151 [arXiv: 1209.0716] [INSPIRE].

[7] NOvA collaboration, B.O'Sheg Oshinowo et al., Survey of the NOvA detectors at Fermilab, FERMILAB-CONF-13-466-AD-PPD, Fermilab, Batavia U.S.A. (2013) [inSPIRE].

[8] MINOS collaboration, Neutrino oscillation physics at Fermilab: the NuMI-MINOS project, NUMI-L-375, Fermilab, Batavia U.S.A. (1998) [INSPIRE].

[9] MINOS collaboration, J.L. Thron, The MINOS long-baseline neutrino oscillation experiment, NUMI-216, Fermilab, Batavia U.S.A. (1996), pg. 1245 [INSPIRE].

[10] V. Papadimitriou et al., Design of the LBNE beamline, FERMILAB-CONF-14-181-AD, Fermilab, Batavia U.S.A. (2014) [INSPIRE].

[11] B. Batell, M. Pospelov and A. Ritz, Exploring portals to a hidden sector through fixed targets, Phys. Rev. D 80 (2009) 095024 [arXiv:0906.5614] [INSPIRE].

[12] P. deNiverville, M. Pospelov and A. Ritz, Observing a light dark matter beam with neutrino experiments, Phys. Rev. D 84 (2011) 075020 [arXiv:1107.4580] [InSPIRE].

[13] P. deNiverville, D. McKeen and A. Ritz, Signatures of sub-GeV dark matter beams at neutrino experiments, Phys. Rev. D 86 (2012) 035022 [arXiv: 1205.3499] [INSPIRE].

[14] B. Batell, P. deNiverville, D. McKeen, M. Pospelov and A. Ritz, Leptophobic dark matter at neutrino factories, Phys. Rev. D 90 (2014) 115014 [arXiv: 1405.7049] [InSPIRE].

[15] MiniBooNE collaboration, R. Dharmapalan et al., Low mass WIMP searches with a neutrino experiment: a proposal for further MiniBooNE running, arXiv:1211.2258 [INSPIRE].

[16] J. Goodman et al., Constraints on light Majorana dark matter from colliders, Phys. Lett. B 695 (2011) 185 [arXiv: 1005.1286] [INSPIRE]. 
[17] Y. Bai, P.J. Fox and R. Harnik, The Tevatron at the frontier of dark matter direct detection, JHEP 12 (2010) 048 [arXiv: 1005.3797] [INSPIRE].

[18] J. Goodman et al., Constraints on dark matter from colliders, Phys. Rev. D 82 (2010) 116010 [arXiv: 1008.1783] [INSPIRE].

[19] P.J. Fox, R. Harnik, J. Kopp and Y. Tsai, Missing energy signatures of dark matter at the LHC, Phys. Rev. D 85 (2012) 056011 [arXiv:1109.4398] [INSPIRE].

[20] J. Goodman and W. Shepherd, LHC bounds on UV-complete models of dark matter, arXiv: 1111.2359 [INSPIRE].

[21] H. An, X. Ji and L.-T. Wang, Light dark matter and $Z^{\prime}$ dark force at colliders, JHEP 07 (2012) 182 [arXiv:1202.2894] [INSPIRE].

[22] P.J. Fox, R. Harnik, R. Primulando and C.-T. Yu, Taking a razor to dark matter parameter space at the LHC, Phys. Rev. D 86 (2012) 015010 [arXiv:1203.1662] [INSPIRE].

[23] H. An, R. Huo and L.-T. Wang, Searching for low mass dark portal at the LHC, Phys. Dark Univ. 2 (2013) 50 [arXiv:1212.2221] [INSPIRE].

[24] P. Fayet, Constraints on light dark matter and $U$ bosons, from $\psi, \Upsilon, K^{+}, \pi^{0}, \eta$ and $\eta^{\prime}$ decays, Phys. Rev. D 74 (2006) 054034 [hep-ph/0607318] [INSPIRE].

[25] P. Fayet, $U$-boson production in $e^{+} e^{-}$annihilations, $\psi$ and $\Upsilon$ decays and light dark matter, Phys. Rev. D 75 (2007) 115017 [hep-ph/0702176] [INSPIRE].

[26] P. Fayet, Invisible $\Upsilon$ decays into light dark matter, Phys. Rev. D 81 (2010) 054025 [arXiv:0910.2587] [INSPIRE].

[27] G.K. Yeghiyan, $\Upsilon$ decays into scalar dark matter, arXiv:0910.2071 [INSPIRE].

[28] A. Badin and A.A. Petrov, Searching for light dark matter in heavy meson decays, Phys. Rev. D 82 (2010) 034005 [arXiv: 1005.1277] [INSPIRE].

[29] M.L. Graesser, I.M. Shoemaker and L. Vecchi, A dark force for baryons, arXiv:1107.2666 [INSPIRE].

[30] I.M. Shoemaker and L. Vecchi, Unitarity and monojet bounds on models for DAMA, CoGeNT and CRESST-II, Phys. Rev. D 86 (2012) 015023 [arXiv:1112.5457] [INSPIRE].

[31] M. Reece and L.-T. Wang, Searching for the light dark gauge boson in GeV-scale experiments, JHEP 07 (2009) 051 [arXiv: 0904.1743] [INSPIRE].

[32] J.D. Bjorken, R. Essig, P. Schuster and N. Toro, New fixed-target experiments to search for dark gauge forces, Phys. Rev. D 80 (2009) 075018 [arXiv: 0906.0580] [INSPIRE].

[33] R. Essig, P. Schuster, N. Toro and B. Wojtsekhowski, An electron fixed target experiment to search for a new vector boson $A^{\prime}$ decaying to $e^{+} e^{-}$, JHEP 02 (2011) 009 [arXiv:1001.2557] [INSPIRE].

[34] R. Essig, R. Harnik, J. Kaplan and N. Toro, Discovering new light states at neutrino experiments, Phys. Rev. D 82 (2010) 113008 [arXiv: 1008.0636] [INSPIRE].

[35] E. Izaguirre, G. Krnjaic, P. Schuster and N. Toro, New electron beam-dump experiments to search for MeV to few-GeV dark matter, Phys. Rev. D 88 (2013) 114015 [arXiv:1307.6554] [INSPIRE].

[36] D.E. Morrissey and A.P. Spray, New limits on light hidden sectors from fixed-target experiments, arXiv:1402.4817 [INSPIRE]. 
[37] E. Izaguirre, G. Krnjaic, P. Schuster and N. Toro, Physics motivation for a pilot dark matter search at Jefferson laboratory, Phys. Rev. D 90 (2014) 014052 [arXiv:1403.6826] [INSPIRE].

[38] A.E. Nelson and N. Tetradis, Constraints on a new vector boson coupled to baryons, Phys. Lett. B 221 (1989) 80 [INSPIRE].

[39] C.D. Carone and H. Murayama, Possible light U(1) gauge boson coupled to baryon number, Phys. Rev. Lett. 74 (1995) 3122 [hep-ph/9411256] [INSPIRE].

[40] C.D. Carone and H. Murayama, Realistic models with a light U(1) gauge boson coupled to baryon number, Phys. Rev. D 52 (1995) 484 [hep-ph/9501220] [INSPIRE].

[41] B.A. Dobrescu and F. Yu, Coupling-mass mapping of dijet peak searches, Phys. Rev. D 88 (2013) 035021 [arXiv:1306.2629] [INSPIRE].

[42] M. Duerr, P. Fileviez Perez and M.B. Wise, Gauge theory for baryon and lepton numbers with leptoquarks, Phys. Rev. Lett. 110 (2013) 231801 [arXiv: 1304.0576] [INSPIRE].

[43] CDF collaboration, T. Aaltonen et al., A search for dark matter in events with one jet and missing transverse energy in p $\bar{p}$ collisions at $\sqrt{s}=1.96 \mathrm{TeV}$, Phys. Rev. Lett. 108 (2012) 211804 [arXiv: 1203.0742] [INSPIRE].

[44] ATLAS collaboration, Search for new phenomena with the monojet and missing transverse momentum signature using the ATLAS detector in $\sqrt{s}=7 \mathrm{TeV}$ proton-proton collisions, Phys. Lett. B 705 (2011) 294 [arXiv:1106.5327] [INSPIRE].

[45] ATLAS collaboration, Search for dark matter candidates and large extra dimensions in events with a jet and missing transverse momentum with the ATLAS detector, JHEP 04 (2013) 075 [arXiv:1210.4491] [INSPIRE].

[46] CMS collaboration, Search for dark matter and large extra dimensions in monojet events in pp collisions at $\sqrt{s}=7 \mathrm{TeV}$, JHEP 09 (2012) 094 [arXiv: 1206.5663] [INSPIRE].

[47] CMS collaboration, Search for dark matter, extra dimensions and unparticles in monojet events in proton-proton collisions at $\sqrt{s}=8 \mathrm{TeV}$, arXiv:1408.3583 [INSPIRE].

[48] BaBAR collaboration, B. Aubert et al., A search for invisible decays of the $\Upsilon(1 S)$, Phys. Rev. Lett. 103 (2009) 251801 [arXiv:0908.2840] [INSPIRE].

[49] BES collaboration, M. Ablikim et al., Search for the invisible decay of $J / \psi$ in $\psi(2 S) \rightarrow \pi^{+} \pi^{-} J / \psi$, Phys. Rev. Lett. 100 (2008) 192001 [arXiv:0710.0039] [INSPIRE].

[50] R. Essig, J. Mardon, M. Papucci, T. Volansky and Y.-M. Zhong, Constraining light dark matter with low-energy $e^{+} e^{-}$colliders, JHEP 11 (2013) 167 [arXiv:1309.5084] [INSPIRE].

[51] CRESST-II collaboration, G. Angloher et al., Results on low mass WIMPs using an upgraded CRESST-II detector, Eur. Phys. J. C 74 (2014) 3184 [arXiv: 1407.3146] [INSPIRE].

[52] DAMIC collaboration, J. Barreto et al., Direct search for low mass dark matter particles with CCDs, Phys. Lett. B 711 (2012) 264 [arXiv:1105.5191] [INSPIRE].

[53] SuPERCDMS collaboration, R. Agnese et al., Search for low-mass weakly interacting massive particles using voltage-assisted calorimetric ionization detection in the SuperCDMS experiment, Phys. Rev. Lett. 112 (2014) 041302 [arXiv: 1309.3259] [INSPIRE].

[54] D.P. Finkbeiner, S. Galli, T. Lin and T.R. Slatyer, Searching for dark matter in the CMB: a compact parameterization of energy injection from new physics, Phys. Rev. D 85 (2012) 043522 [arXiv: 1109.6322] [INSPIRE]. 
[55] S. Galli, F. Iocco, G. Bertone and A. Melchiorri, Updated CMB constraints on dark matter annihilation cross-sections, Phys. Rev. D 84 (2011) 027302 [arXiv:1106.1528] [INSPIRE].

[56] G. Hutsi, J. Chluba, A. Hektor and M. Raidal, WMAP7 and future CMB constraints on annihilating dark matter: implications on GeV-scale WIMPs, Astron. Astrophys. 535 (2011) A26 [arXiv: 1103.2766] [INSPIRE].

[57] T. Lin, H.-B. Yu and K.M. Zurek, On symmetric and asymmetric light dark matter, Phys. Rev. D 85 (2012) 063503 [arXiv:1111.0293] [INSPIRE].

[58] K.M. Zurek, Asymmetric dark matter: theories, signatures and constraints, Phys. Rept. 537 (2014) 91 [arXiv: 1308.0338] [INSPIRE].

[59] C. Boehm and P. Fayet, Scalar dark matter candidates, Nucl. Phys. B 683 (2004) 219 [hep-ph/0305261] [INSPIRE].

[60] R. Essig, E. Kuflik, S.D. McDermott, T. Volansky and K.M. Zurek, Constraining light dark matter with diffuse X-ray and gamma-ray observations, JHEP 11 (2013) 193 [arXiv: 1309.4091] [INSPIRE].

[61] G. Steigman, B. Dasgupta and J.F. Beacom, Precise relic WIMP abundance and its impact on searches for dark matter annihilation, Phys. Rev. D 86 (2012) 023506 [arXiv:1204.3622] [INSPIRE].

[62] J. Alwall, M. Herquet, F. Maltoni, O. Mattelaer and T. Stelzer, MadGraph 5: going beyond, JHEP 06 (2011) 128 [arXiv:1106.0522] [InSPIRE].

[63] N.D. Christensen and C. Duhr, FeynRules - Feynman rules made easy, Comput. Phys. Commun. 180 (2009) 1614 [arXiv:0806.4194] [INSPIRE].

[64] MINOS collaboration, P. Adamson et al., Active to sterile neutrino mixing limits from neutral-current interactions in MINOS, Phys. Rev. Lett. 107 (2011) 011802 [arXiv: 1104.3922] [INSPIRE].

[65] D.E. Soper, M. Spannowsky, C.J. Wallace and T.M.P. Tait, Scattering of dark particles with light mediators, Phys. Rev. D 90 (2014) 115005 [arXiv: 1407.2623] [INSPIRE].

[66] MiniBoonE and MINOS collaborations, P. Adamson et al., First measurement of $\nu_{\mu}$ and $\nu_{e}$ events in an off-axis horn-focused neutrino beam, Phys. Rev. Lett. 102 (2009) 211801 [arXiv: 0809 . 2447] [INSPIRE].

[67] MicroBooNE collaboration, T. Katori, MicroBooNE, a liquid argon time projection chamber (LArTPC) neutrino experiment, AIP Conf. Proc. 1405 (2011) 250 [arXiv:1107.5112] [INSPIRE].

[68] C. Admas et al., LAr1-ND: testing neutrino anomalies with multiple LAr TPC detectors at Fermilab, FERMILAB-PROPOSAL-1053, Fermilab, Batavia U.S.A. (2013) [InSPIRE].

[69] LBNE collaboration, C. Adams et al., The Long-Baseline Neutrino Experiment: exploring fundamental symmetries of the universe, arXiv:1307.7335 [INSPIRE]. 